\title{
The Full Outline of UnResponsiveness score is more efficient than the Glasgow Coma Scale in the prognosis of stroke
}

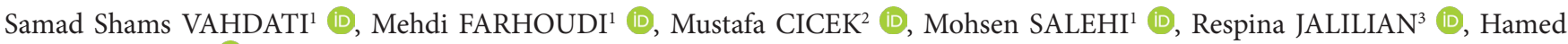 \\ HOJJATPANAH ${ }^{3}$ [D
}

\begin{abstract}
Neuroscience Research Center, Imam Reza Medical Center, Tabriz University of Medical Sciences, Tabriz, Iran
Department of Emergency Medicine, School of Medicine, Karadeniz Technical University, Trabzon, Turkey

Tabriz University of Medical Sciences, Tabriz, Iran
\end{abstract}

Corresponding Author: Hamed HOJJATPANAH

E-mail: hamedh94.55@gmail.com

Submitted: 18.06.2019 Accepted: 09.08.2019

\begin{abstract}
Objectives: After being a solid method of measuring consciousness for decades, the Glasgow Coma Scale (GCS) was offered to be replaced with a better option: The Full Outline of Unresponsiveness (FOUR) score, which several studies later confirmed to be superior due to its independence from verbal response and ability to consider brain stem reflexes. In this study, we decided to compare the two scales in the prognosis of stroke, which has never been conducted before.

Patients and Methods: We conducted both methods on 150 stroke patients during admission to the emergency service and assessed them during a 3-month follow-up after discharge.

Results: The type of stroke was $80 \%$ ischemic and $20 \%$ hemorrhagic. $55.3 \%$ survived after a 3-month follow-up. The FOUR score had a significant converse relation with hospitalization, a relation with the GCS lacked. The FOUR score also had a stronger correlation with the outcome of stroke than the GCS. Additionally, the receiver operating characteristic (ROC) curve showed greater sensitivity and specificity in the FOUR score in the prognoses of stroke patients.

Conclusion: We recommend using the FOUR score as the first priority.

Keywords: Glasgow Coma Scale, Full Outline of Unresponsiveness score, Stroke, Consciousness, Emergency medicine
\end{abstract}

\section{INTRODUCTION}

Stroke is the third most common cause of disability and the second most common cause of death worldwide. This condition can cause a wide variety of symptoms, one of which is loss of consciousness. Any neural damage due to hypoxia of a cerebrovascular accident can reduce the level of consciousness. There exist variable tools for measurement and assessment of consciousness [1].The Glasgow Coma Scale (GCS) has still remained as a common and simple method that is used in several health care centers, even the small ones, and can be provided easily by all clinicians, even general practitioners. It is scored by three items: eye opening, motor response and verbal response [2]. There is also another scale for evaluating the level of consciousness. The Full Outline of UnResponsiveness (FOUR) score has recently gained attention, which is composed of four clinically distinct categories of evaluation: eye reaction, motor function, brainstem reflexes and the respiratory pattern [3]. This scale has demonstrated superiorities to the GCS, leading clinicians to utilize it for more confidence and accuracy [4].

Discovering the prognosis of stroke in different patients at initial moments can help the doctors to make a reliable decision and perform an appropriate management $[5,6]$. Different factors influence the prognosis of stroke and finding scales that show outcomes of patients with the most simple and fast way and highest possible accuracy is a challenge these days. Several studies have been performed to evaluate the prognosis of stroke and outcomes due to initial onsets and several scales used for this purpose.

In this study, we investigate using the GCS and FOUR score versus the other tools and also compare each other in order to evaluate the loss of consciousness due to stroke, and predict the outcomes of stroke by these scales, seeking to further assess the superiority of the FOUR score relative to the GCS.

\section{PATIENTS and METHODS}

\section{Study Design}

In this cross-sectional study, after receiving approval ( approval number: 5/4/3858), from the Ethics Committee in Research, Tabriz University of Medical Sciences, Tabriz, Iran, the selection

How to cite this article: Vahdati SS, Farhoudi M, Cicek M, Salehi M, Jalilian R, Hojjatpanah H. The Full Outline of UnResponsiveness score is more efficient than the Glasgow Coma Scale in the prognosis of stroke. Marmara Med J 2019; 32: 97-101. doi: 10.5472/marumj.637561 
criteria were established as follows: Inclusion criteria: all patients with signs of stroke attending the emergency department between April 2013 and March 2014. All of these patients underwent biomedical imaging after being included in the study and the diagnosis was established in all cases.

Exclusion criteria: patients with serum electrolyte disorders, patients with any abnormalities in blood glucose level, patients with diagnoses apart from stroke, patients who received anesthesia, neuromuscular blockers, any sedative agents such as : midazolam, morphine, fentanyl, sufentanil, pancuronium bromide, atracurium besylate, propofol, within the past 48 hours, patients with hearing impairment and/or muteness were excluded.

\section{Implementation}

The sample size was calculated by the Morgan's Table, requiring 384 patients in a citywide population of 1.5 million with a $5 \%$ Margin of Error. All the patients were randomly selected. The patients' next of kin obtained written assurances of information confidentiality and provided consent with the study. The patients were assessed during the first hours after admission to the emergency room. Emergency specialists were the investigators who performed the assessment during admission and before examining the patients. All emergency specialists were re-educated of the procedures of calculation and the criteria of inclusion and exclusion. The physicians graded the patients, according to both the GCS and FOUR score at the time of arrival, and evaluated the patients' levels of consciousness.

The demographics and other information recorded for each patient were as follows: age, sex, the FOUR score and the GCS score at the time of arrival and date of discharge, prognosis of each patient according to the modified Rankin Scale (MRS), which was used to measure the degree of disability in patients who have had a stroke and included morbidity, duration of hospitalization and mortality during discharge from the emergency department. All the patients were followed up according to the mRS questionnaire, which continued for three months. Forty-four patients were missed during the follow-up. Additionally, adjusted regression models were calculated for possible confounders; and the mRS and/or the incidence of death were noted as well.

\section{Statistical analysis}

Eventually, the relation between the GCS and FOUR scores at the time of arrival was reviewed and compared with the prognosis and outcome during discharge according to the mRS. The data was collected and analysed using the SPSS software version 21.0 (IBM Corporation, Armonk, NY, USA). A discriptive study was run for demographic data (mean, mode, median) and the Pearson correlation coefficient (PCC) was used in order to find associations between the prognoses, mean hospital durations, mRS and each rating scale. The p-value below 0.05 was considered meaningful.

\section{RESULTS}

A total of 150 patients participated in the study: 66 males (44\%) and 84 females (56\%). The mean age was 69.79 \pm 1.11 (Min=21,
Max=91). The type of stroke was ischemic in 120 patients $(80 \%)$ and hemorrhagic in the remaining 30 (20\%). The means of the FOUR score and the GCS at arrival time were $13.92 \pm$ $0.23(\operatorname{Min}=3, \operatorname{Max}=16)$ and $11.73 \pm 0.25(\min =3, \max =13)$, respectively. The mean duration of hospitalization was $14.95 \pm$ 1.52 days $(\mathrm{Min}=1, \operatorname{Max}=110)$.

Forty-five patients (30\%) died in hospital, 22 patients $(14.7 \%)$ died after discharge and 83 patients (55.3\%) survived after a 3-month follow-up. The results of the GCS and FOUR score at arrival, the MRS score at discharge and after the follow-up are provided in Tables I and II.

Table I. The FOUR score and the GCS at the time of arrival compared with the MRS at discharge (the data are presented as Mean \pm SE)

\begin{tabular}{|l|l|l|}
\hline GCS & FOUR score & MRS \\
\hline $13 \pm 2$ & $16 \pm 0.001$ & 0 (no symptoms) \\
\hline $13.75 \pm 1.25$ & $15.5 \pm 0.5$ & 1 \\
\hline $14.8 \pm 0.42$ & $16 \pm 1.01$ & 2 \\
\hline $13.83 \pm 0.61$ & $15.67 \pm 0.22$ & 3 \\
\hline $13.13 \pm 0.36$ & $15.29 \pm 0.21$ & 4 \\
\hline $10.72 \pm 0.43$ & $13.72 \pm 0.35$ & 5 \\
\hline $9.95 \pm 0.45$ & $11.43 \pm 0.51$ & 6 (deceased) \\
\hline$<0.001$ & $<0.001$ & p value \\
\hline
\end{tabular}

GCS: Glasgow Coma Scale, FOUR: Full Outline of UnResponsiveness, MRS: Modified Rankin Scale SE: standard error

Table II. The FOUR score and the GCS at the time of arrival compared with the MRS after a three-month follow-up (the data are presented as Mean \pm SE)

\begin{tabular}{|l|l|l|}
\hline FOUR score & GCS & MRS \\
\hline $13.8 \pm 0.62$ & $15.7 \pm 0.21$ & 0 (no symptoms) \\
\hline $13.75 \pm 0.42$ & $15.71 \pm 0.16$ & 1 \\
\hline $13.85 \pm 0.37$ & $15,69 \pm 0.17$ & 2 \\
\hline $13.47 \pm 0.44$ & $15.27 \pm 0.24$ & 3 \\
\hline $11.69 \pm 0.83$ & $14.38 \pm 0.53$ & 4 \\
\hline $11 \pm 3$ & $14.5 \pm 1.5$ & 5 \\
\hline $10.52 \pm 0.52$ & $13.56 \pm 0.44$ & 6 (deceased ) \\
\hline$<0.001$ & $<0.001$ & p value \\
\hline
\end{tabular}

GCS: Glasgow Coma Scale, FOUR: Full Outline of Unresponsiveness, MRS: Modified Rankin Scale SE: standard error

The FOUR score of the stroke patients had a significant converse association with hospitalization ( $\mathrm{p}$-value $=0.01, \mathrm{PCC}=-0.2$ ). However, the GSC of the stroke patients had no significant association with hospitalization ( $\mathrm{p}$-value $=0.1, \mathrm{PCC}=-0.13$ ).

The results of the ROC curve (Figure 1) for investigating sensitivity and specificity of the GCS and FOUR scores in the prognoses of the patients showed a significant relation between the FOUR scores and the prognosis : cut off point $=9.5$, sensitivity $=100 \%$, specificity $=82.1 \%$, and curved surface $=-0.84$ ( $\mathrm{p}$-value $<0.001$ ). In addition, it showed a significant relation between the GCS and the prognosis: cut off point $=10.5$, sensitivity $=85.5 \%$, specificity $=68.2 \%$ and curved surface $=-0.81$ (p-value $<0.001$ ). 


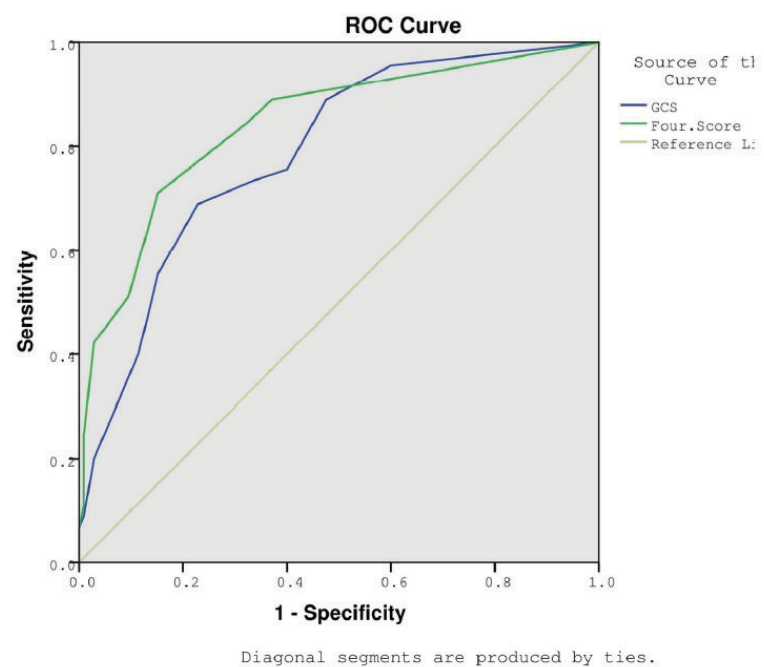

Figure 1. The receiver operating characteristic (ROC) curve for a comparison of the GCS and FOUR score in the prognosis of stroke

\section{DISCUSSION}

The GCS is the procedure of scoring by eye opening, motor response and verbal response and the FOUR score consists of eye reaction, motor function, brainstem reflexes and the respiratory pattern. The GCS is a simple and quickly computable method. Although, it is used widely in hospitals as a standard scale for scoring the level of consciousness [7, 8], it has defects and limitations:

1. A very important question is whether it is beneficial to use the verbal response for evaluating level of consciousness. The verbal response is investigated according to orientation in time and place, and requires a complete orientation in order to achieve the full score. But, is it necessary to be completely oriented to time and space to be considered a conscious person? We believe that confusion or stimulation or even fear or emotional stress can reduce the score of level of consciousness in the GCS evaluating. There are also other situations where a patient with no or little verbal response can lose score in measuring level of consciousness such as aphasia due to stroke. In addition, calculating the GCS score in intubated patients is more difficult than calculating the FOUR score. Hence, the latter is more appropriate in intubated patients and in Intensive Care Units (ICUs) $[9,10]$.

2. Another important point is that the GCS lacks the ability to evaluate brain stem reflexes and eye movements or complicated motor responses. Versus, the FOUR score can evaluate brain stem and pons reflexes and yield beneficial information about their situation $[11,12]$.

In the recent years, several studies had pointed the defects of the GCS $[12,13]$. Considering these limitations, the FOUR score is at a higher level. It is not dependant on verbal response while it utilizes brainstem reflexes. The FOUR score can have a poor prognosis on brain death but it can differentiate the locked-in syndrome from a coma. Not only is it as easy as the GCS to utilize, but can also be used at ICUs, and thus has a wider range of usability, higher accuracy and more reliability. It has four items each ranging from 0 to 4 (Table III), whereas the GCS has three items, having 4-6 scores (Table IV). Therefore, calculation is easier in the FOUR score, reducing the probability of errors as a result $[14,15]$.

Table III. Glasgow Coma Scale (GCO)

\begin{tabular}{|l|}
\hline Eye opening \\
\hline 4=spontaneous \\
\hline 3=due to speech \\
\hline 2=due to pain \\
\hline 1=no response \\
\hline Best motor response \\
\hline 6=obedience \\
\hline 5=localization \\
\hline 4=withdrawal \\
\hline 3=abnormal flexion \\
\hline 2=extention \\
\hline $1=$ no response \\
\hline Best verbal response \\
\hline 5=oriented \\
\hline 4=confused conversation \\
\hline 3=inappropriate words \\
\hline 2=incomprehensible sounds \\
\hline 1=no response \\
\hline
\end{tabular}

Table IV. The Full Outline of UnResponsiveness (FOUR) score

Eye response

$4=$ eyelids open or opened, tracking, or blinking to command

$3=$ eyelids open but not tracking

$2=$ eyelids closed but opened due to loud voice

$1=$ eyelids closed but opened due to pain

$0=$ eyelids remain closed notwithstanding pain

\section{Motor response}

4=thumbs-up, fist, or peace sign

$3=$ localizing toward pain area

$2=$ flexion response to pain

$1=$ extension response to pain

$0=$ no response to pain or generalized

myoclonus status

Brainstem reflexes

$4=$ present papillary and corneal reflexes

$3=$ one pupil wide and fixed

$2=$ absent papillary or corneal reflexes

$1=$ absent papillary and corneal reflexes

$0=$ absent papillary, corneal, and cough reflexes

\section{Respiration}

$4=$ not intubated, regular respiration

$3=$ not intubated, Cheyne-Stokes respiration

$2=$ not intubated, irregular respiration

$1=$ above ventilator rate

$0=$ at ventilator rate or apnea 
We performed this study to determine a simple and fast way to make the prognosis of stroke patients and able to guide physicians to make suitable decisions. Determination of the outcome of stroke patients by the GCS or FOUR score is a new study that has never conducted before; and it provided us with a significant relation and showed its reliability. The outcomes of stroke patients were scored by the MRS (Table V) at the time of discharge and 3 months later, showing not only a good correlation between the GCS and the MRS, but also a high prognostic value of the FOUR score in patients with cerebrovascular attacks. Moreover, using this new coma scale might overwhelm the limitations of the GCS at the time of admission to an emergency department. It has also demonstrated advantages at evaluation immediately after cardiopulmonary resuscitation (CPR).

Table V. Modified Rankin Scale (MRS)

\begin{tabular}{|l|}
\hline $0=$ No symptoms at all \\
$\begin{array}{l}\text { 1=Significant disability notwithstanding symptoms; able to carry out all } \\
\text { usual duties and activities }\end{array}$ \\
\hline $\begin{array}{l}\text { 2=Slight disability; unable to carry out all previous activities, but able to run } \\
\text { one's own affairs without assistance }\end{array}$ \\
\hline $\begin{array}{l}\text { 3=Moderate disability; requiring some help, but able to walk without } \\
\text { assistance }\end{array}$ \\
\hline $\begin{array}{l}\text { 4=Moderately severe disability; unable to walk without assistance and unable } \\
\text { to attend to own bodily needs without assistance }\end{array}$ \\
\hline $\begin{array}{l}\text { 5=Severe disability; bedridden, incontinent and requiring constant nursing } \\
\text { care and attention }\end{array}$ \\
\hline 6= Deceased \\
\hline
\end{tabular}

According to our results, both of the scales were equally useful in long-term prediction of the clinical condition, evaluated by the mRS. The FOUR score and the MRS demonstrated an inverse correlation which was even stronger than that between the GCS and MRS $(-0.84$ vs. $-0.81 ; \mathrm{p}<0.001)$.

Wijdicks et al., studied the reliability of the FOUR score for the first time [14]. They have demonstrated that the FOUR score can provide more neurologic information, such as brainstem abnormalities, breathing disorder or brain herniation. The FOUR score and the MRS showed an inverse correlation which was even stronger than that between the GCS and MRS (-0. 547 vs. $-0.514 ; \mathrm{p}<0.001)$. A similar difference of correlation was also seen after the three-month follow up (-0.509 vs. - 0.486). Besides, in patients with stroke, it is capable of detecting the locked-in syndrome.

Kocak et al., have investigated the predictive validity of the FOUR score in acute stroke patients in ICU [3]. The follow-up study of the admitted patients favoured our results. Our findings support the hypothesis that the FOUR score could be regarded superior to the GCS in assessing the prognosis and level of consciousness in patients with acute stroke.

Saika et al., studied on 138 patients in 2015 to investigate the prediction validity of the GCS and FOUR score in patients with brain injury [16]. The mean score of GCS and FOUR was 9.5 (3-11) and 11 (0-16), respectively. The data showed that the mean GCS and FOUR score finding were significantly lower in expired patients. The FOUR score had a cut off point of $7.97 \%$ sensitivity, 97.5\% specificity and a minister curved surface of 0.97. The GCS had a cut off point of $6.98 .3 \%$ sensitivity, $82.4 \%$ specificity and a minister curved surface of -0.95 . Hence, they mentioned that one could use both the GSC and FOUR score to predict the outcome of brain injuries.

Sadaka et al., performed a study on 51 patients in 2011 and distinguished a significant relation between the two scales and the prognoses determined by the MRS in brain injuries [9]. In this study, the minister curved surface that showed hospital mortality was equal to 0.93 for the FOUR score and 0.89 for the GCS. In addition, the minister curved surface for mortality after 3-6 months was equal to 0.85 for the FOUR score and 0.83 for the GCS. Authors pointed that the FOUR score had supremacy to the GCS at predicting the outcome of brain injuries.

There exist several other studies that show the validity of the FOUR score in predicting different brain injuries and its superiority to the GCS. Recently, Stead et al., Iyer et al. and Bruno et al., reported similar results along with others and our results are in line with these observations [12, 17-21].

We have also demonstrated an improvement in the function of the patients, corresponding to a decrease in the follow-up MRS, with a promotion in both the GCS and FOUR score grading. These results may be descriptive of the therapeutic utility in patients with acute stroke. In terms of distinction of the ischemic or hemorrhagic type of stroke, our results were unable to show a significant correlation with the MRS. It would thus be preferable to combine these prognostic indicators in a model with other components of stroke if they were to be used in patient management. The follow-up period was relatively short in our study. We suggest further studies with larger samples and longer follow-ups.

Both the FOUR score and the GCS are appropriate scales for evaluating level of consciousness in stroke patients and in addition, both have a significant relation with prognoses of stroke patients at the time of discharge and the follow-up. As a result, the GCS and FOUR score are useful predictors of the outcome of stroke and can help physicians to make decisions on patient management during initial hours.

Because of the limitations of the GCS due to dependence on verbal response and failure to consider brainstem reflexes, and additionally a stronger significant correlation between the FOUR score and the outcome of stroke according to the MRS, and also a significant association between the FOUR score and hospitalization in our study, we suggest utilizing the FOUR score instead of the GCS. The FOUR score is a reliable and valuable scale in predicting prognosis in patients wth stroke.

Conflict of interest: The authors have no conflicts of interest to declare.

\section{REFERENCES}

[1] Girard TD, Pandharipande PP, Ely EW. Delirium in the intensive care unit. Crit Care 2008;12 Suppl 3:S3. doi: 10.1186/ cc6149 
[2] Marino PL. Marino's the ICU Book. Philadelphia: Lippincott Williams and Wilkins, 2013.

[3] Kocak Y, Ozturk S, Ege F, Ekmekci H. A useful new coma scale in acute stroke patients: FOUR score. Anaesth Intensive Care 2012;40:131-6. doi: 10.1177/0310057X120.400.0115

[4] Chen B, Grothe C, Schaller K. Validation of a new neurological score (FOUR Score) in the assessment of neurosurgical patients with severely impaired consciousness. Acta Neurochir (Wien) 2013 ;155:2133-9. doi: 10.1007/s00701.013.1854-2

[5] Koennecke HC, Belz W, Berfelde D, et al. Factors influencing in-hospital mortality and morbidity in patients treated on a stroke unit. Neurology 2011;77:965-72. doi: 10.1212/ WNL.0b013e31822dc795

[6] Saver JL, Altman H. Relationship between neurologic deficit severity and final functional outcome shifts and strengthens during first hours after onset. Stroke 2012;43:1537-41. doi: 10.1161/STROKEAHA.111.636928

[7] Fischer M, Ruegg S, Czaplinski A, et al. Inter-rater reliability of the Full Outline of UnResponsiveness score and the Glasgow Coma Scale in critically ill patients: a prospective observational study. Crit Care 2010;14:R64. doi: 10.1186/cc8963

[8] Wijdicks EF, Rabinstein AA, Bamlet WR, Mandrekar JN. FOUR score and Glasgow Coma Scale in predicting outcome of comatose patients: a pooled analysis. Neurology 2011 5;77:84-5. doi: 10.1212/WNL.0b013e318220ac06

[9] Sadaka F, Patel D, Lakshmanan R. The FOUR score predicts outcome in patients after traumatic brain injury. Neurocrit Care 2012;16:95-101. doi: 10.1007/s12028.011.9617-5

[10] Idrovo L, Fuentes B, Medina J, et al. Validation of the FOUR Score (Spanish Version) in acute stroke: an interobserver variability study. Eur Neurol 2010;63:364-9. doi: $10.1159 / 000292498$

[11] Eken C, Kartal M, Bacanli A, Eray O. Comparison of the Full Outline of Unresponsiveness Score Coma Scale and the Glasgow Coma Scale in an emergency setting population. Eur J Emerg Med 2009;16:29-36. doi: 10.1097/ MEJ.0b013e32830346ab

[12] Stead LG, Wijdicks EF, Bhagra A, et al. Validation of a new coma scale, the FOUR score, in the emergency department. Neurocrit Care 2009;10:50-4. doi: 10.1007/s12028.008.9145-0
[13] Zappa S, Fagoni N, Bertoni M, et al. Determination of imminent brain death using the Full Outline of Unresponsiveness Score and the Glasgow Coma Scale: A prospective, multicenter, pilot feasibility study. J Intensive Care Med 2017 Jan 1:885.066.617738714. doi: 10.1177/088.506.6617738714

[14] Wijdicks EF, Bamlet WR, Maramattom BV, Manno EM, McClelland RL. Validation of a new coma scale: The FOUR score. Ann Neurol 2005;58:585-93. 10.1002/ana.20611

[15] Kornbluth J, Bhardwaj A. Evaluation of coma: a critical appraisal of popular scoring systems. Neurocrit Care 2011;14:134-43. doi: 10.1007/s12028.010.9409-3

[16] Saika A, Bansal S, Philip M, Devi BI, Shukla DP. Prognostic value of FOUR and GCS scores in determining mortality in patients with traumatic brain injury. Acta Neurochir (Wien) 2015;157:1323-8. doi: 10.1007/s00701.015.2469-6

[17] Iyer VN, Mandrekar JN, Danielson RD, Zubkov AY, Elmer JL, Wijdicks EF. Validity of the FOUR score coma scale in the medical intensive care unit. Mayo Clin Proc 2009;84:694-701. doi: 10.1016/S0025-6196(11)60519-3

[18] Bruno MA, Ledoux D, Lambermont B, et al. Comparison of the Full Outline of UnResponsiveness and Glasgow Liege Scale/Glasgow Coma Scale in an intensive care unit population. Neurocrit Care 2011;15:447-53. doi: 10.1007/ s12028.011.9547-2

[19] McNett M, Amato S, Gianakis A, et al. The FOUR score and GCS as predictors of outcome after traumatic brain injury. Neurocrit Care 2014;21:52-7. doi: 10.1007/s12028.013.9947-6

[20] Sepahvand E, Jalali R, Mirzaei M, Ebrahimzadeh F, Ahmadi M, Amraii E. Glasgow Coma Scale versus Full Outline of UnResponsiveness Scale for prediction of outcomes in patients with traumatic brain injury in the Intensive Care Unit. Turk Neurosurg 2016;26:720-4. doi: 10.5137/1019-5149. JTN.13536-14.0

[21] Kasprowicz M, Burzynska M, Melcer T, Kübler A. A comparison of the Full Outline of UnResponsiveness (FOUR) score and Glasgow Coma Score (GCS) in predictive modelling in traumatic brain injury. Br J Neurosurg 2016;30:211-20. doi: 10.3109/02688.697.2016.1161173 